\title{
Synthesis of mesoporous silica nanoparticles by means of a hydrogel
}

\author{
Abdolraouf Samadi-Maybodi ${ }^{*}$ and Amir Vahid
}

\begin{abstract}
Synthesis and application of mesoporous silicate nanoparticles are important areas of research in many fields such as drug delivery, medicine, catalysis, and optic. The method of synthesis strongly affects the properties of a product. In this work, the mesoporous silica nanoparticles were synthesized by means of a hydrogel. The obtained product was characterized by X-ray diffraction, scanning electron microscopy, and nitrogen physisorption. The results show that highly ordered mesoporous silica nanoparticles were synthesized by means of a hydrogel.
\end{abstract}

Keywords: Hydrogel, Mesoporous silica nanoparticles, Nanoparticles, Synthesis

\section{Background}

Since the discovery of a new family of mesoporous materials, i.e., M-41 s [1,2], they have attracted growing attention in scientific research areas. Their potential in many fields such as catalysis [3], separation [4], optic [5], medicine [6], biology [7], and biotechnology [8] was investigated by many groups. In some areas such as optic, drug delivery [9], medicine, biology, and biotechnology, mesoporous silica nanoparticles (MSNs) are very important. Mesoporous silica could be synthesized in different shapes and morphologies, including thin films, fibers, and particles in various forms and sizes $[10,11]$. So far, there have been many reports in the synthesis of mesoporous silica nanoparticles using various strategies and reactants. The most common strategies for the preparation of MSNs are (1) stopping particle growth at early stages (high dilution technique), (2) encapsulation by a second surfactant system, (3) aerosolbased process, (4) confined space synthesis, and (5) reduced condensation speeds. Each one of the abovementioned methods has advantages and disadvantages [12-15]. Hydrogels were also used for the synthesis of zeolite nanocrystals [16]. However, an appropriate synthesis must be resulted in small, easily dispersed, and wellordered silica particles.

\footnotetext{
* Correspondence: samadi@umz.ac.ir

Analytical Division, Faculty of Chemistry, Mazandaran University, Babolsar
} 47416-95447, Iran

\section{望

\section{Methods}

Materials and apparatus

Cetylmethylammonium bromide (CTAB), tetraethylorthosilicate (TEOS), aqueous ammonia $(25 \% \mathrm{w} / w)$, and 2hydroxymethylcellulose were all purchased from Merck (Whitehouse Station, NJ, USA). Deionized water was used as a solvent. All chemicals were used as purchased, and no further purification was performed.

Powder X-ray diffraction (XRD) patterns of synthesized samples were recorded on a Seifert TT 3000 diffractometer (Ahrensburg, Germany) using $\mathrm{Cu} \mathrm{K} \alpha$ radiation of wavelength $0.15405 \mathrm{~nm}$. Diffraction data were recorded between 1 and $10^{\circ} 2 \theta$ with a resolution of $0.01^{\circ} 2 \theta$ with the scan rate of $0.12 \theta / \mathrm{min}$. Scanning electron micrographs were recorded using a Zeiss DSM 962 (Carl Zeiss, Inc., Oberkochen, Germany). The samples were deposited on a sample holder with an adhesive carbon foil and sputtered with gold. Adsorption-desorption isotherms of nitrogen were obtained at $77 \mathrm{~K}$ using a BELSORP instrument (BEL Japan, Inc., Toyonaka-shi, Japan). The samples were outgassed at $423 \mathrm{~K}$ and 1 $\mathrm{mPa}$ for $4 \mathrm{~h}$ prior to measurement was performed. The yield of the method was defined as the weight of the final product (which assumed is only $\mathrm{SiO}_{2}$ ) per weight of the $\mathrm{SiO}_{2}$ (equivalent with TEOS) in the initial mixture.

\section{Synthesis}

In a typical synthesis, $2.6 \mathrm{~g}$ of CTAB was added to $100 \mathrm{~g}$ of deionized water and stirred to give a clear solution. Then $10.5 \mathrm{~g}$ of ammonia solution (25 wt.\%) was added 


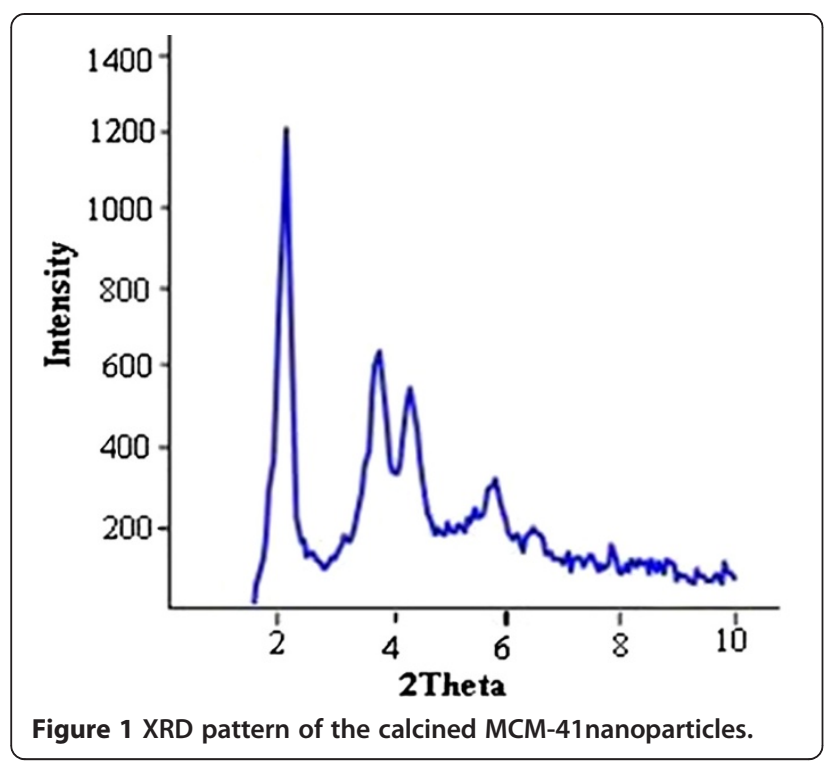

to this solution. Then, $10 \mathrm{~g}$ of TEOS was added to this solution. At the end of this step, $20 \mathrm{~g}$ of 2hydroxymethylcellulose solution (15 wt.\%) was added to the suspension. The obtained final suspension was stirred for about $4 \mathrm{~h}$ followed by 2 days aging in oven at $373 \mathrm{~K}$. Afterwards, the precipitate was filtered rather than centrifuged, washed with deionized water, and dried at $373 \mathrm{~K}$ overnight. The filtering step was slow and may have taken several minutes. The obtained product was placed in a muffle furnace and calcined with a heating rate of $1 \mathrm{~K} / \mathrm{min}$ to $823 \mathrm{~K}$ and held at this temperature for $5 \mathrm{~h}$ under air.

\section{Results and discussion}

In the synthesis medium of MCM-41 nanoparticles, 2-hydroxymethylcellulose has a key role in the production of MCM-41 particles in the nanometer range. It is well

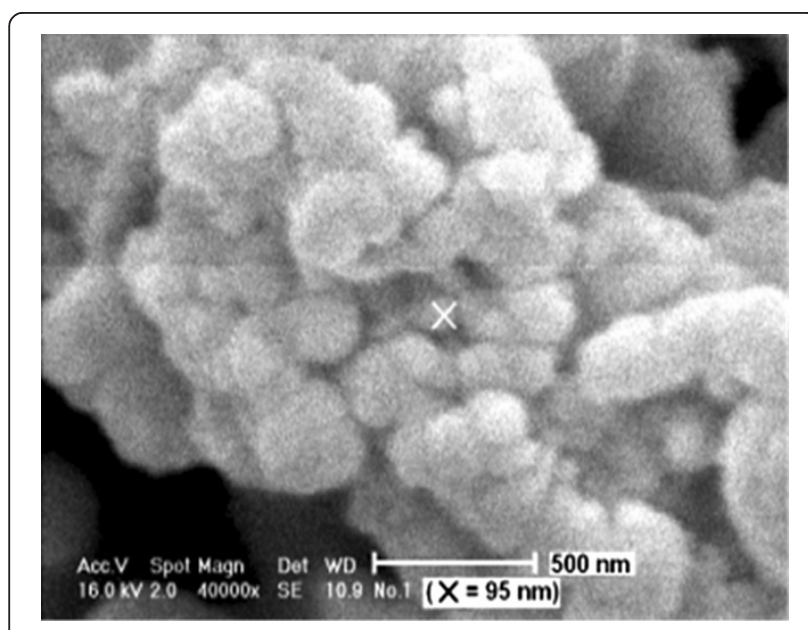

Figure 2 SEM micrograph of MCM-41 nanoparticles.

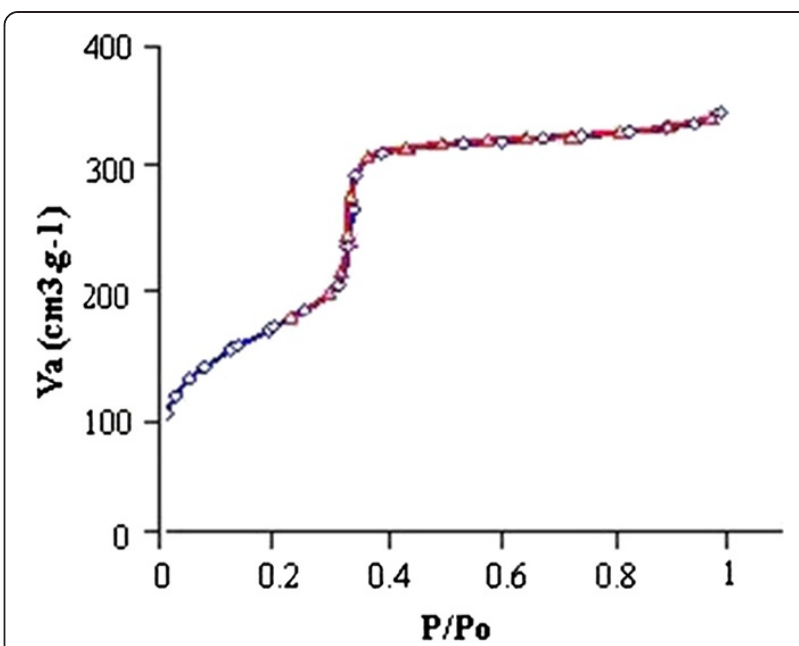

Figure $3 \mathrm{~N}_{2}$ adsorption/desorption isotherm of the MCM-41 nanoparticles at $\mathbf{7 7} \mathrm{K}$. Blue and red lines correspond to the adsorption and desorption branches, respectively.

known that 2-hydroxymethylcellulose constructs a threedimensional cage-like networks (hydrogel) in the synthesis medium which can act as nanoreactors for the synthesis of MSNs $[17,18]$. This phenomenon prevents free diffusion of performed seeds and crystallites of MCM-41 and favors the production of nanoparticles rather than the agglomerated large particles. The XRD pattern of the calcined sample is shown in Figure 1. It can be seen that there are at least five well-defined brag peaks which specify the formation of highly ordered MSNs. The XRD pattern also revealed that although thermal calcination was applied for the removal of both CTAB and 2-hydroxymethylcellulose from the as-synthesized sample, the final product still remains highly ordered. This implies that the final product is very stable against thermal treatment. Another remarkable

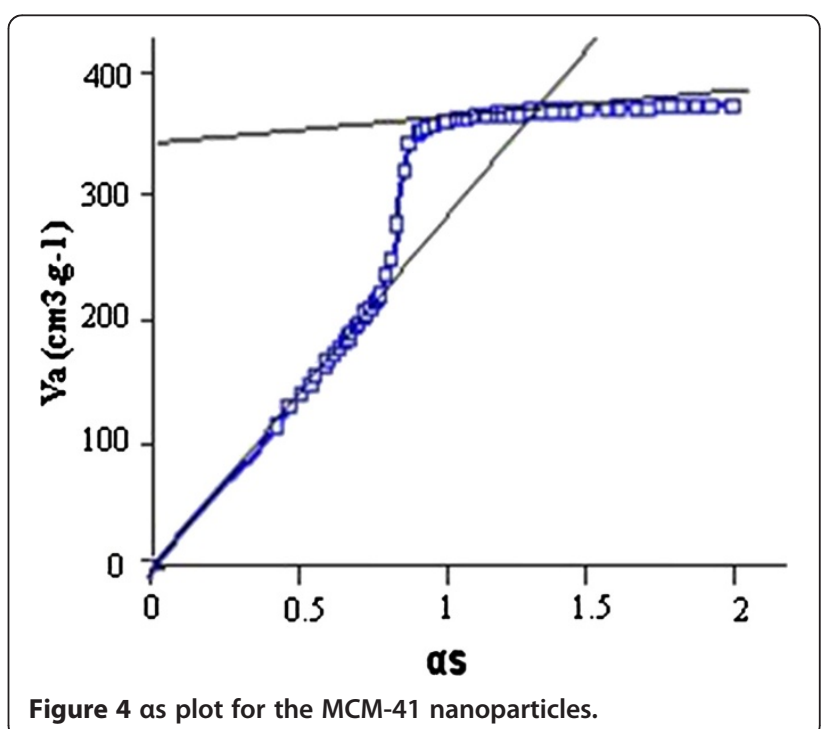


Table 1 Textural properties of the calcined MCM-41 nanoparticles

\begin{tabular}{lcccc}
\hline$S_{\mathrm{BET}}\left(\mathrm{m}^{2} / \mathbf{g}\right)^{\mathrm{a}}$ & $\boldsymbol{V}_{\boldsymbol{t}}\left(\mathrm{cm}^{3} / \mathbf{g}\right)^{\mathrm{b}}$ & $\boldsymbol{a}(\mathbf{n m})^{\mathrm{c}}$ & $\boldsymbol{W}_{\boldsymbol{d}}(\mathbf{n m})^{\mathbf{d}}$ & $\boldsymbol{b}_{\boldsymbol{d}}(\mathbf{n m})^{\mathrm{e}}$ \\
\hline 608 & 0.53 & 5.1 & 3.91 & 1.38 \\
\hline
\end{tabular}

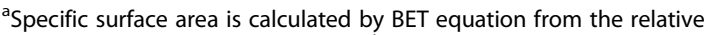
adsorption range from 0.05 to 0.25 ; ${ }^{b}$ total pore volume; ${ }^{c}$ lattice parameter obtained from XRD diffractogram $\left(2 d_{100} / \sqrt{ } 3\right)$; ${ }^{d}$ geometrical pore diameter calculated by pressure-independent method: $W_{d}=c d_{100}\left[\rho \mathrm{V}_{\mathrm{p}} /\left(1+\rho \mathrm{V}_{\mathrm{p}}\right)\right]^{1 / 2}$ where $W_{d}$ is the pore diameter, $c$ is equal to $1.213, \rho$ is the silica density $\left(2.20 \mathrm{~cm}^{3} / \mathrm{g}\right)$, and $V_{p}$ is the mesopore volume which can be obtained either from as-plot or top of the step of mesopore filling [19]; 'Wall thickness obtained by lattice parameter minus the distance between midpoints of the hexagonal cross section (the latter value is equal to $\mathrm{W}_{d} / 1.05$ ).

result that has not been reported by now is the intensity of second and third peaks which are about one half of the d100 peak (see Figure 1). This is an indication of high structural order in the obtained product.

Figure 2 illustrates the SEM micrograph of the synthesized MCM-41 nanoparticles. The order of particle size is ranged from about 60 to $350 \mathrm{~nm}$. The results obtained from this experiment indicate that the yield of this method is very high up to $95 \%$ by weight.

Adsorption-desorption isotherm of nitrogen that illustrated in Figure 3 shows a type IV isotherm which is the characteristic of mesoporous materials according to the IUPAC classification [19]. The adsorption and desorption branches of the isotherm coincide each other indicating reversible adsorption and desorption of the nitrogen inside the mesopores. The sharp inflection in the capillary condensation step at which the gas uptake suddenly occurs over a narrow relative pressure ranges from 3.2 to 3.8 specifying that the MCM-41 nanoparticles have a very narrow pore size distribution. After the capillary condensation step, the long plateau at higher relative pressures with a low increase in the amount of adsorbed gas shows that the mesopores are filled at low relative pressure approximately at 0.34 . The $\alpha$ s plot in Figure 4 provides a comparison between adsorption behavior of the obtained MCM-41 nanoparticles with a known reference material, i.e., macroporous silica. Lack of any deviation from linearity at low values of $\alpha$ s or, in other words, at low pressures indicates the absence of microporosity. Upward deviation which occurs at higher $\alpha$ s values is due to the capillary condensation in primary mesopores.

At higher pressures, i.e., near to the saturation vapor pressure $(\alpha s>2)$, there is very low deviation from the linearity which is due to the low amount of adsorbed gas in secondary mesopores and external surface of MCM-41 nanoparticles. High surface area which was calculated from Brunauer-Emmett-Teller (BET) equation together with the last mentioned results approve that high quality MCM-41 nanoparticles are synthesized. The textural properties of the calcined sample are given in Table 1.

\section{Conclusions}

In this research, we report a novel method for the synthesis of MCM-41 nanoparticles in a one-pot procedure using 2-hydroxymethylcellulose as a hydrogel. There is no any increase in the reaction volume compared with the other methods which applied for the synthesis of the bulky MCM-41 material. Furthermore, due to the use of inexpensive and nonhazardous hydrogel, i.e., 2-hydroxymethylcellulose, this method is applicable, straightforward, and easy for the large scale synthesis of MSNs for industrial use. The yield of this method is not only very high up to $95 \%$ by weight but also results in highly ordered MCM-41 nanoparticles.

\section{Competing interests}

The authors declare that they have no competing interests.

\section{Authors' contributions}

AS edited the manuscript ad provided valuable guides for the writing of the whole manuscript. AV carried out synthesis and charactrization of MCM-41 nanoprticles. Both authors read and approved the final manuscrpt.

\section{Acknowledgment}

We are grateful to the Materials \& Energy Research Center of Karaj for performing nitrogen adsorption measurement.

Received: 28 January 2011 Accepted: 27 February 2012

Published: 24 May 2013

\section{References}

1. Kresge, CT, Leonowicz, ME, Roth, WJ, Vartuli, JC, Beck, JS: Nature 359, 710 (1992)

2. Yanagisawa, T, Shimizu, T, Kuroda, K, Kato, C: Bull. Chem. Soc. Jpn. 63, 988 (1990)

3. Taguchi, A, Schuth, F: Micropor. Mesopor. Mater. 77, 1 (2005)

4. Grun, M, Kurganov, AA, Schacht, S, Schuth, F, Unger, KK: J. Chromatogr. A 740, 1 (1996)

5. Zanjanchi, MA, Ebrahimian, A, Alimohammadi, Z: Opt. Mater. 29, 794 (2007)

6. Lu, J, Liong, M, Zink, Jl, Tamanoi, F: Small 8, 1341 (2007)

7. Slowing, II, Trewyn, BG, Lin, VSY: J. Am. Chem. Soc. 129, 8845 (2007)

8. Torney, F, Trewyn, BG, Lin, VSY: Nature Nanotech. 2, 295 (2007)

9. Slowing, II, Vivero-Escoto, JL, Wu, CW, Lin, VSY: Adv. Drug Delivery Rev. 60, 1278 (2008)

10. Wan, Y: Zhao, DY: Chem. Rev. 107(2), 821 (2007)

11. Pevzner, S, Regev, O, Yerushalmi-Rozen, R: Curr. Opin. Colloid Interface Sci. 4, 420 (1999)

12. Fowler, CE, Khushalani, D, Lebeau, B, Mann, S: Adv. Mater. 13, 649 (2001)

13. Cai, Q, Luo, ZS, Pang, WQ, Fan, YW, Chen, XH, Cui, FZ: Chem. Mater. 13, 258 (2001)

14. Rathousky, J, Zukalova, M, Kooyman, PJ, Zukal, A: Colloids Surf. A 241, 81 (2004)

15. Nooney, Rl, Thirunavukkarasu, D, Chen, Y, Josephs, R, Ostafin, AE: Chem. Mater. 14, 4721 (2002)

16. Wang, H, Holmberg, BA, Yan, Y: J. Am. Chem. Soc. 125, 9928 (2003)

17. Cho, EC, Kim, JW, Fernández-Nieves, A, Weitz, DA: Nano Lett. 8, 168 (2008)

18. Yui, N, Park, P, Mrsny, RJ: Reflexive Polymers and Hydrogels. CRC press, Portland OR, USA (2004)

19. Kruk, M, Jaroniec, M: J. Phys. Chem. B 104, 292 (2000)

\section{doi:10.1186/2228-5326-3-39}

Cite this article as: Samadi-Maybodi and Vahid: Synthesis of mesoporous silica nanoparticles by means of a hydrogel. International Nano Letters 2013 3:39. 